\title{
Language Profiles in ASD, SLI, and ADHD
}

\author{
Hilde M. Geurts · Mariëtte Embrechts
}

Published online: 3 June 2008

(C) The Author(s) 2008

\begin{abstract}
Developmental disorders might differ in their language profiles when using parent reports. The first study indicated that school aged children with ASD have similar language profiles as children with ADHD. Both groups had relatively more difficulties with pragmatics than with structural language aspects. The second study indicated that both preschoolers with ASD and those with SLI show the opposite pattern, thus having relatively more difficulties with structural language aspects than with pragmatics. Finally, an increase in the presence of ADHD characteristics of impulsivity in these preschoolers is associated with an increase in language difficulties, while there is no such relation with inattention. It seems useful to evaluate the communication abilities of children regularly in the course of development and take ADHD characteristics into account. Finally recommendations on clinical use of the Children's Communication Checklist-2 (CCC-2, Bishop 2003) are discussed.
\end{abstract}

Keywords Pragmatics - CCC-2 - ADHD - ASD · SLI $\cdot$ Language $\cdot$ Communication

H. M. Geurts ( $\square)$

Department of Psychonomics, University of Amsterdam, Roetersstraat 15, 1018 WB Amsterdam, The Netherlands e-mail: h.m.geurts@uva.nl

M. Embrechts

ENT Department - Children Audiology Center, University

Medical Center St Radboud, Nijmegen, The Netherlands

\section{Introduction}

Within communication the form, content, and use of language are all three essential ingredients. Deficits in the last ingredient, pragmatics or the appropriate use of language within social and situational contexts (Martin and McDonald 2003; Rapin 1996; Tannock and Schachar 1996) have been observed in a broad range of developmental disorders (e.g., Bishop 1998; Norbury et al. 2004; Gilmour et al. 2004; Towbin et al. 2005). The precise nature and extent of these so called pragmatic difficulties seem to differ among the specific diagnostic groups. Pragmatic language use refers to a broad array of social linguistic skills. Hence, pragmatic difficulties can be present in the domain of the communicative intention, presupposition, or discourse management (Landa 2005). Exactly these types of communication difficulties are at the core of the autism spectrum disorders (ASD). Children with ASD are characterized by communication impairments, social impairments and restricted, stereotypical patterns of behavior and interests (American Psychiatric Association [APA] 2000; Volkmar et al. 2004). The focus of the current paper will be the communication difficulties that children with ASD encounter.

\section{Communication Difficulties in ASD}

Children with ASD are often delayed in linguistic areas such as lexical and syntactic knowledge, phonology and morphology, although these impairments are less prominent than the impairments in the pragmatic aspects of language (Lord and Paul 1997). The difficulties with language and communication are already present early in life (Landa 2007; Rapin and Dunn 2003). However, it seems that the pragmatic impairments remain life long whereas the other language related difficulties are no longer 
manifest in every single child when they mature (Rapin and Dunn 2003). Studies focusing on preschoolers with ASD revealed that at this age the preschoolers encounter deficits in the form, content, and use of language. As when they grew older the pragmatics difficulties are the most profound (Rapin and Dunn 2003). In typically developing children pragmatic competence precedes the competence in language form as these children are already able to communicate a long time before they use their first words. However, deficits in language components such as syntax, morphology, and phonology can also affect pragmatic competence (see for an overview Bara et al. 1999). Children who have language problems before the age of three appear to be at very high risk for developing ASD in early childhood (Miniscalco et al. 2006). If language impairments at the age of five persist into adolescence than these early language impairments are predictive for attention and social difficulties in adolescence (Snowling et al. 2006). Expressive language problems seem to be more associated with difficulties in attention, while the combination of receptive and expressive language difficulties were more associated with social difficulties (Snowling et al. 2006). This suggests that different profiles of language skills and deficits are associated with specific deficiencies in other developmental domains later in life.

\section{Communication Difficulties in ADHD}

Language disorders are often present in children with attention deficit hyperactivity disorder (ADHD; e.g., Baird et al. 2000; Bruce et al. 2006; Oram et al. 1999; Tannock and Schachar 1996). However, the cardinal features of ADHD are inattentiveness, hyperactivity, and impulsivity (APA 2000) and language related impairments are not required to fulfill criteria of ADHD. Bishop and Baird (2001) showed that children with ADHD had pragmatic difficulties: children with ADHD showed more stereotyped conversations, had more problems with conversational rapport, and demonstrated more problems with social relationships compared to typically developing children, who show no deviances in their language development. A striking finding was that children with ADHD did hardly differ from children with ASD. These observed pragmatic difficulties in ADHD children had been replicated in two other studies (both described in Geurts et al. 2004), but the pragmatic difficulties in children with ADHD were, in general, less profound as those observed in children with ASD. The studies (Bishop and Baird 2001; Geurts et al. 2004) differed in the assessment of the children, but the children in both studies were from a very broad age range (5-17 years), and both applied the same questionnaire to study the language profiles of these children, the Children's Communication Checklist (CCC; Bishop 1998).
The CCC is a questionnaire that encompasses several aspects of language, including language form (such as syntax) and pragmatics. Numerous studies showed that by using this questionnaire one can obtain reliable and valid language profiles (e.g., Bishop 1998; Bishop and Baird 2001; Botting and Conti Ramsden 1999), but the CCC has been revised. The successor, the CCC-2 (Bishop 2003) contains 10 scales: four related to different aspects of the pragmatic use of language; four assessing structural aspects of language use; and two scales assessing nonlanguage domains that were included to obtain information about other autistic features that are not directly related to language (see Table 1 and description of the material in Study 1). The CCC-2 differs from the original version in a number of ways and these adaptations might affect the specific outline of the differences and similarities in the observable language profiles with the CCC-2 in developmental disorders.

\section{Goals}

In order to study language profiles with the CCC-2 we carried out two studies. The goal of the first study was to replicate the CCC language profiles in children with ASD and ADHD aged 7-14 years with the CCC-2. Based on the earlier studies (Bishop 1998; Geurts et al. 2004) we hypothesize that school aged children with ADHD will have scores in between typically developing children and children with ASD. Moreover, we expect that independent of diagnosis the children will have relatively more language deficiencies related to pragmatics than to language form (see also Rapin and Dunn 2003; Mawhood et al. 2000).

The goal of the second study is to explore whether the profile of language skills and difficulties in preschoolers with ASD is similar to the language profile in children with ASD aged 7-14 years of age. We hypothesize that preschoolers (4-7 years) with ASD will encounter difficulties in all language domains. These difficulties will be equal across pragmatics and language form. In older ASD children (7-14 years) we expect mainly the presence of pragmatic problems.

\section{Communication Difficulties in SLI}

To be able to determine whether there is a specific ASD related language profile in preschoolers a comparison with another clinical group is essential. The language disorders seen in ASD largely overlap with the language disorders observed in children with specific language impairments (Bishop 2001; Kjelgaard and Tager Flusberg 2001). Specific language impairment (SLI) is a diagnosis given to children with specific language difficulties that cannot be 


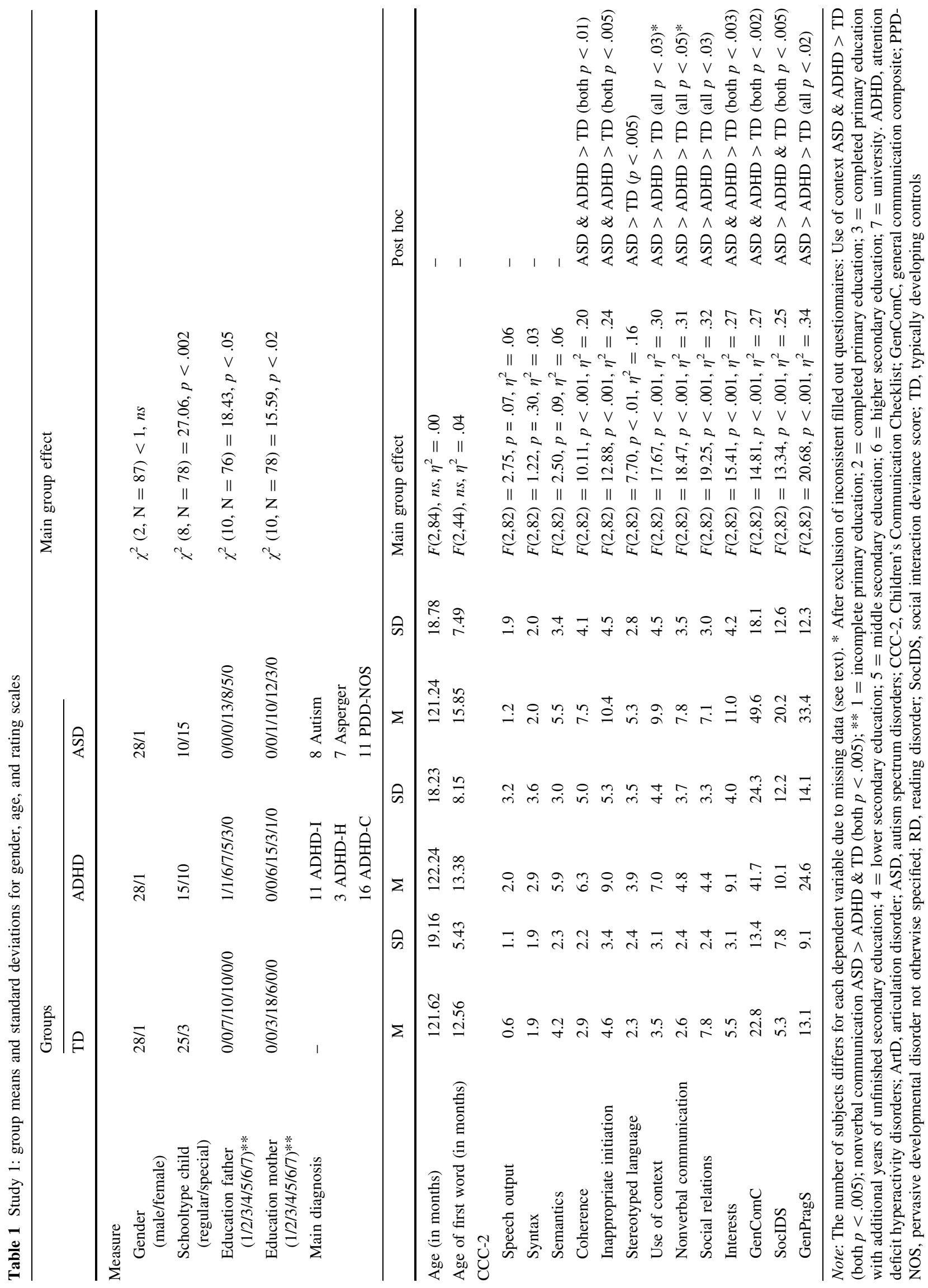


explained by other factors. These children might encounter deficits in structural components of language, but could also have specific pragmatic disorders which can exist in the absence of a structural language disorders per se (Adams and Lloyd 2005; Bishop and Norbury 2002; Botting 2003). A direct comparison of children with ASD with children with SLI on the CCC-2 (Norbury et al. 2004) revealed that children with ASD exhibit more profound pragmatic difficulties as compared to children with SLI. Moreover, in the SLI group the structural language impairments were disproportional to the pragmatic/social difficulties, while the opposite pattern was present in the ASD group (Norbury et al. 2004). However, the children in this study were all aged between 8 and 14 years. As in the second study we focus on preschoolers, we hypothesize that children with ASD and SLI will show a similar pattern of pragmatic and structural language impairments, but that children with ASD will show relatively more difficulties at the two scales tapping into autistic like behavior (i.e., social relationships and interests).

So, the present paper presents two different studies in which children with several different clinical diagnoses within diverse age groups were compared on the CCC-2. In Study 1, children with ASD will be directly compared to children with ADHD and typically developing (TD) children (all aged 7-14 years). In Study 2, a direct comparison will be made between preschoolers with ASD and preschoolers with SLI (all aged 4-7 years). The first goal of these two studies was to explore whether there is a specific ASD language profile in terms of the nature and extent of their language skills and deficits and whether this profile depends on the age range. The second goal was to explore how this ASD language profile is related to the language profiles in other disorders such as ADHD and SLI.

\section{Method Study 1}

\section{Participants}

Two clinical groups participated in this study: (a) an ASD group and (b) an ADHD group. CCC-2 data were gathered for 87 children with a clinical diagnosis with ages in between 7 and 14 years. Children were recruited from University outpatient clinics, Child and Youth Psychiatric Hospitals, and Special Educational Services for children with ADHD and ASD. Only those children meeting the following criteria were admitted: (1) a prior independent diagnosis of ADHD or ASD by the child's health care professional (the diagnostic classification was based on diagnostic assessment by a child psychiatrist and multiple informants (i.e., speech-specialists, psychologists, and educationalists); (2) being a native Dutch speaker; (3) no neurological, sensory, or motor impairment; (4) no hearing impairment (threshold was $25 \mathrm{~dB}$ ); 5) no extreme language deprivation or unfavorable language environment; and (6) no mental retardation. The ADHD group consisted of 30 children, but the data was incomplete for one child and four parents were inconsistent in their answers ${ }^{1}$ on the CCC-2. The ASD group consisted of 57 children and 13 parents were inconsistent in their answers. The two groups were finally matched on consistency in answering, gender, and age (in months). Hence, 29 children were included in each of the clinical groups.

TD children were recruited from regular schools throughout the Netherlands to participate in a normative study of the CCC-2. From these data $(n=1795)$, data of 29 children were selected to serve as a TD comparison group. The TD children were matched for gender and age with the ASD group. All parents were consistent in their answers and none of these children had problems in school or showed signs of any developmental problem according to their parents.

Most of the clinicians, involved in the clinical assessment of the children participating in the ASD group, filled out a list of questions concerning the specific diagnosis of these children (45 clinicians returned their form of which 26 were included in the final ASD group, see above). Unfortunately only 6 clinicians returned the questionnaire regarding the assessment of the children with ADHD. The other clinicians were called upon, so based on these telephone calls we verified the clinical diagnosis of the remaining children. For details regarding the diagnosis see Table 1.

\section{Material}

The CCC-2 (Bishop 2003; Dutch translation: Geurts 2007) was developed to measure various aspects of communicative impairments. The questionnaire covers (besides language structure skills) mainly pragmatic skills, which are necessary in social communication. The CCC- 2 contains 70 items that are grouped in 10 scales with 7 items/ scale: (A) speech; (B) syntax; (C) semantics; (D) coherence; (E) inappropriate initiation, (F) stereotyped language, (G) use of context; (H) nonverbal communication; (I) social relationships, (J) interests. In addition, a general communication composite (GenComC) may be calculated, which is an overall measure of communication skills and consists of the sum of the scores on scales A-H. The second composite score that may be calculated is the social interaction deviance score (SocIDS) which is a difference

\footnotetext{
$\overline{1}$ In the CCC -2 manual (Bishop 2003, p. 7) there is a short description on how one can calculate the internal consistency of the answers of the parents.
} 
score between the sum of scale E, H, I, and J and the sum of scale A, B, C, and D. The third composite score is the general pragmatics score (GenPragS) that is the sum of the scores on scales D-H. This composite score, giving an overall impression of the pragmatic abilities, is comparable with the composite score of the original CCC (Bishop 1998). Items are scored on a four point scale (less than once a week [or never], at least once a week, but not every day, once or twice a day, and several times [more than twice] a day [or always]). Of each of the scales five items are difficulty items (negatively formulated items) and two items are strength items (positively formulated items). The higher the score on the CCC-2, the more impaired the child is.

Reliability of the CCC-2 was examined in children 4-15 years of age and parents served as informants. The internal consistency $(\alpha)$ ranged from .66 to $.80(n= \pm 535)$ and the inter-rater reliability $(r)$ between parents and teachers ranged from .16 to $.53(n=55$; Bishop 2003). The inter-rater reliability for the GenComC was .40 and for the SocIDS .79. The GenPragS was not included in the English version of the CCC-2. The CCC-2 was translated into Dutch using a two-way translation procedure and reliability measures were re-examined in a Dutch group of children aged 4-16 years with again parents as informants. The internal consistency $(\alpha)$ ranged from .53 to .75 $(n= \pm 2,575)$ and the test-retest reliability $(\rho)$ ranged from .49 to $.77(n=55$; Geurts 2007) in TD children. In clinical samples the internal consistency $(\alpha)$ ranged from .48 to .88 (4-7 years of age $n= \pm 50$; 7-15 years of age $n= \pm 58$ ). The internal consistency for the GenComC ranged from .82 to .89 and for the GenPragS from .78 to .88 .

\section{Procedure}

An information letter, an informed consent form, and a copy of the CCC-2 were sent to all parents of the children of five different schools. In the seven participating clinics this package was sent to children who could be included based on the aforementioned criteria. In total we have sent 416 packages for Study 1 and Study 2 to the schools and clinics and $51 \%$ of these questionnaires were returned. In addition, information was obtained from the parents concerning the child's mental or physical handicaps, the child's language development, family language background, and education of parents. The education of the parents was rated on an ordinal scale with a number from 1 to 7 , in which 1 means that a person did not complete primary school and 7 meaning that a person has a university degree. After a written informed consent was obtained from the parents, a questionnaire regarding the assessment details was send to the clinician(s) of children with a clinical diagnosis. The study was approved upon by the ethical committee of the Psychology Department of the University of Amsterdam.

\section{Statistical Analyses}

Group differences for the CCC-2 scales were analyzed using MANOVAs with group (three levels) as a between factor.

\section{Results Study 1}

The results for the three groups on the CCC-2 are shown in Table 1. As expected, the groups did neither differ with respect to age nor with respect to gender. Hence, the groups were successfully matched. Moreover, the groups did not differ with respect to the time at which they uttered their first word. Please note that there was a large number of missing data points here. Apparently a lot of parents $(n=40)$ could not recall when their child uttered the first word or first sentence. However, the groups did differ in the educational backgrounds of the parents. More parents of the children with ASD had a higher educational background compared to the parents of the other two groups (ADHD and TD).

\section{Communication Profiles in ADHD, ASD, and TD Children Aged 7-14 years}

As expected, there was a main effect of group across the scales, Wilk's $\Lambda=0.50, F(20,146)=3.04, p<.001$, $\eta^{2}=.29$. Groups differed from each other on seven of the 10 scales (see Table 1). No group differences were found on speech output, syntax, and semantics, all three language form scales. On three scales, use of context, nonverbal communication and social relationships, children with ASD demonstrated more problems than the two other groups, whereas children with ADHD evidenced significant deficiencies compared to TD children. However, for both the use of context scale and the nonverbal communication scale the results altered after the exclusion of the inconsistent reports. Children with ASD and with ADHD still differed from the TD on the use of context scale, but did not differ from each other. On the nonverbal communication scale the ASD group differed from both the ADHD and TD group. On three other scales, Coherence, Inappropriate initiation, and interests both the ASD and ADHD group demonstrated deficiencies compared with the TD group. On the stereotyped language scale only the ASD group showed deficiencies compared to the TD group.

There was also a main effect of group when including the three composite scores as dependent measures, Wilk's $\Lambda=0.59, F(6,160)=7.93, p<.001, \eta^{2}=.23$. On the 
GenComC the ASD group was expected to obtain the highest ratings of all groups-meaning more difficultieswhereas the TD group was hypothesized to obtain the lowest ratings of all groups-implying a better score, so less difficulties. The ADHD group was predicted to obtain ratings in between those of the ASD and TD group. This was not the case. Both children with ASD and children with ADHD had communication difficulties as compared to TD children. However, children with ASD and children with ADHD could not be differentiated from each other on this general communication measure.

All children had a positive score on the second composite score, the SocIDS, indicating relatively higher scores on pragmatics/social scales as compared to language form scales. The scores of the two clinical groups seemed higher than the score of the TD group, but the score of the two clinical groups did neither differ significant from each other nor did the score of the ADHD group differ from the TD group. Children with ASD seem to have more pragmatic/social difficulties as compared to children with ADHD when difficulties in language form have been taken into account. This is in line with the expectations. Also on the GenPragS the children with ASD and children with ADHD could be differentiated from each other. Moreover, both clinical groups had a significantly higher score than the TD children.

\section{Conclusions Study 1}

As expected both school aged children with ASD and children with ADHD encountered pragmatic difficulties. However, only the parents of children with ASD reported that these children indeed have relatively more problems with pragmatics/social skills than with the language structure. On the structural language scales the clinical groups showed no deficiencies. This is in contrast to our former study (Geurts et al. 2004) in which school aged children with ASD did show deficits in language form. At most scales the clinical groups could not be differentiated from each other, although in most cases the ADHD group had scores in between the ASD group and the TD group even though this did not reach significance. However, the clinical groups differed from each other on the use of context, the use of nonverbal communication, and social relationships (but please note that the differences in social relationships was the only robust finding). Children with ASD had clearly more severe problems in these areas than children with ADHD. Difficulties in these areas are crucial to receive a diagnosis of ASD (APA 2000; Volkmar et al. 2004).

The second study was conducted to extend the ASD related findings to preschoolers and to a different population with language disorders (i.e., preschoolers with SLI). As school aged children with ADHD seem to have a similar profile of language skills and deficiencies as children with ASD (Study 1) we also took the presence of ADHD characteristics into account.

One of the diagnostic criteria for ADHD is that the symptoms should be present before the age of seven (APA 2000). However, it is disputable whether the diagnosis of ADHD is already a valid diagnosis in preschoolers because it is difficult to distinguish between clinically significant problem behaviors and those that are transient (Campbell 1995; Marakovitz and Campbell 1998). Based on the Diagnostic Statistical Manual-fourth edition (DSM-IV; APA 2000) it is not evident which specific symptoms should be present and whether they already interfere with the child's language abilities at this young age. Although, there are a number of studies that focus on several cognitive domains in preschoolers with ADHD characteristics (e.g., Marakovitz and Campbell 1998; Thorell and Wahlstedt 2006), we are not aware of studies focusing on language abilities in these preschoolers.

As has been described in the general introduction, the presence of persistent language disorders earlier in life is an important risk factor for the development of psychiatric disorders when reaching middle or late childhood (Miniscalco et al. 2006; Snowling et al. 2006). However, it might be that in these language disordered children the psychiatric disorder, e.g. ADHD characteristics, was already present before the age of six. Therefore, we will study the impact of the presence of ADHD characteristics in preschoolers on their language abilities. We predict that an increase in the presence of ADHD characteristics will lead to an increase in the observed language difficulties.

\section{Method Study 2}

\section{Participants}

Two clinical groups participated in this study: (a) an ASD group and (b) a SLI group. CCC-2 data were gathered for 65 preschoolers with a clinical diagnosis with ages in between 4 and 7 years. Children were recruited from University outpatient clinics, General Health Care Clinics, Special Educational Services for children with Language Impairment (LI) and ASD. The admission criteria for this study were similar to the criteria of Study 1 with one difference: all children needed to have a prior independent diagnosis of LI or ASD by the child's health care professional. The LIdiagnosis was always based on an assessment with standardized tests by a speech and language therapist.

The SLI group consisted of 29 children, but from one child the data were incomplete and five parents were inconsistent 


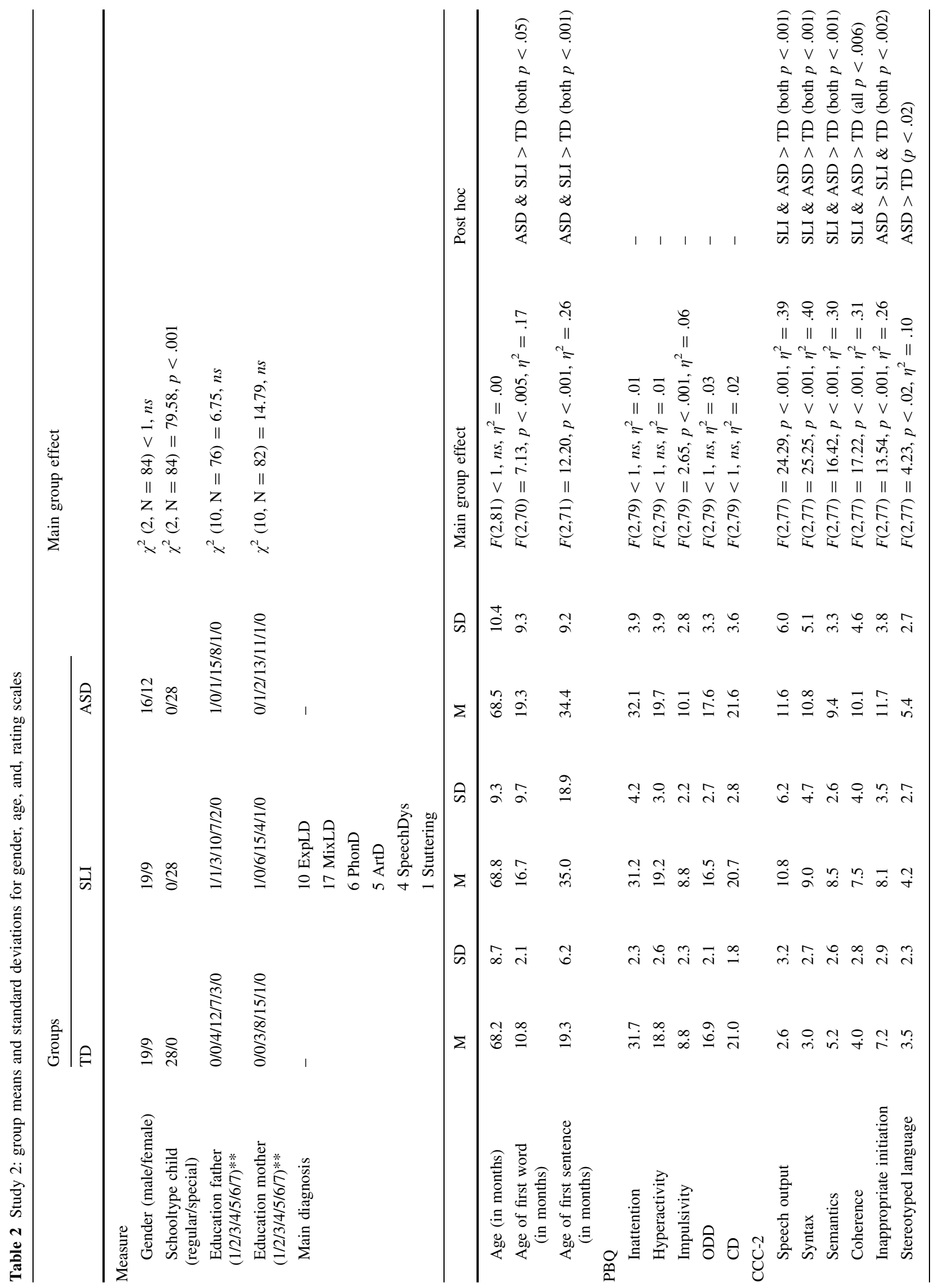




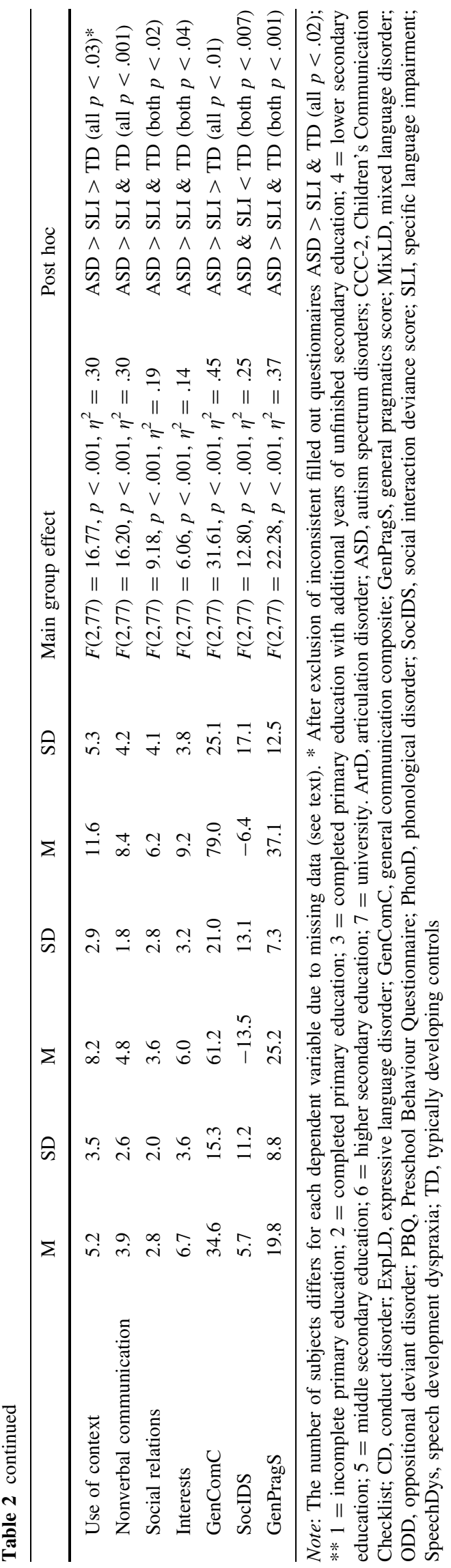

(see footnote 1) in their answers on the CCC-2. The ASD group consisted of 36 children, the data were incomplete for one child and again five parents were inconsistent in their answers. The TD group consisted of 58 preschoolers who were recruited from regular schools. None of these children had problems in school or showed signs of any developmental problems according to their parents. For four of these children the data were incomplete and five parents were inconsistent in their answers. The three groups were finally matched on consistency in answering, gender, and age (in months). This procedure resulted in an SLI group of 28 preschoolers, an ASD group of 28 preschoolers, and a TD group of 28 preschoolers. The clinicians who were involved with the clinical diagnosis of the children participating in the SLI group filled out a list of questions concerning the specific diagnosis of these children. Eighteen of these children had one clinical diagnosis, seven had two clinical diagnoses, and three children had more than two clinical diagnoses (for details please see Table 2). Of only 9 (6 autism and 3 PDDNOS) of the 28 ASD children we also received a completed clinician questionnaire, so of most of these children the ASD diagnosis is confirmed by teacher and parents but not specified by the clinician.

\section{Material}

To determine the communication profile we used the CCC-2, see Study 1. For the purpose to recognize ADHD characteristics as possible predictor for the CCC-2 score we used the Preschool Behaviour Questionnaire (PBQ; Smidts and Oosterlaan 2005). Also this questionnaire was filled out by the parents and consisted of 58 items within six domains: attentional problems, hyperactivity, impulsivity, conduct disorders, oppositional deviant disorder, and other externalizing problems. The first three scales are the so called ADHD scales and the items correspond to the ADHD symptoms listed in the DSM-IV, but are combined with additional items to enhance reliability. Items are scored on a four point scale (does not apply at all, applies to some extent, applies very much, and definitely applies). The internal consistency ranged from .68 to .82 for the scale scores.

Procedure

See Study 1, except that an additional questionnaire on behavior of the child (the PBQ) was sent to the parents.

\section{Statistical Analyses}

First, group differences for the CCC-2 scales were analyzed using MANOVA with group (three levels) as a between factor. To investigate the nature of the group main effects, pair wise group comparisons were conducted with 
alpha levels adjusted for multiple tests (Bonferroni). These analyses were conducted with and without the inclusion of the inconsistent filled out questionnaires. Only when exclusion of the questionnaires that were filled out inconsistently altered the results, this was reported. Please note that in general the results were the same, so the values in Table 2 are with the inclusion of all children.

Second, multiple regression analyses were used to evaluate how ADHD characteristics predicted communication abilities in preschoolers. These analyses were conducted separately for each of the 10 scales and the GenComC and GenPragS of the CCC-2. The influence of the different ADHD characteristics were studied while taking the impact of age into account. The predictors were entered in two steps: First, age of the child was entered because this variable is known to be associated with language abilities. Second, the three different scale scores, inattention, hyperactivity, and impulsivity were entered.

\section{Results Study 2}

The results for the three groups on the CCC-2 are shown in Table 2. Note that the matching was successful as the groups did neither differ with respect to age nor with respect to gender, even thought there were slightly less boys in the ASD group. Moreover the groups did not differ with respect to the educational background of the father and the mother. The preschoolers in the TD group all went to regular school, while most of the children with a clinical diagnosis went to special educational services. According to the information given by the parents the groups differed with respect to the time at which they uttered their first word and their first sentences. Both preschoolers with ASD and with SLI were older than TD preschoolers when they spoke their first word and/or sentences. Again a number of parents $(\mathrm{n}=11)$ could not recall when their child uttered the first word, however most of the parents could recall this developmental milestone.

\section{Communication Profiles in SLI, ASD, and TD}

Preschoolers Aged 4-7 years

As expected, groups differed from each other on each of the 10 scales of the CCC-2, Wilk's $\Lambda=0.40, F(20,136)=3.92$, $p<.001, \eta^{2}=.37$. On one scale, use of context, preschoolers with ASD demonstrated more problems than the two other groups, whereas preschoolers with SLI evidenced significant more problems compared to TD preschoolers. However, the difference between the SLI and the TD group disappeared when excluding the inconsistent data. On four other scales, inappropriate initiation, non-verbal communication, social relationships, and interests the ASD group demonstrated more problems compared to both the SLI and the TD group, while the SLI group could not be differentiated from the TD group. However, on all four structural language scales, speech output, syntax, coherence, and semantics, both clinical groups had higher scores, indicating difficulties, than the TD preschoolers. On the stereotyped language scale preschoolers with ASD had a higher score (indicating more problems) compared to the TD preschoolers, but the comparisons with the SLI group did not reach significance.

The groups differed from each other on the three composite measures, Wilk's $\Lambda=0.45, F(6,150)=12.15$, $p<.001, \eta^{2}=.33$. On the GenComC the ASD group was expected to obtain the highest ratings of all groups, whereas the TD group was hypothesized to obtain the lowest ratings of all groups. The SLI group was predicted to obtain ratings in between those of the ASD and TD group. This was indeed the case as there was a main effect of group (see Table 2) and both preschoolers with ASD and with SLI had communication difficulties as compared to TD preschoolers. Moreover, preschoolers with ASD encountered more communication problems than SLI preschoolers. There was also a significant main effect of group on the second composite score, the SocIDS. In contrast to Study 1, preschoolers with ASD and SLI both had a negative score (indicating more difficulties in structural language components compared to pragmatic/social components), while preschoolers with TD had a positive score. The negative scores of the two clinical groups were significantly lower than the positive score of the TD group, but the score of the two clinical groups did not differ significantly from each other. On the GenPragS preschoolers with ASD obtained a significantly higher score than both preschoolers with SLI and TD preschoolers, indicating that-in comparison-they have the greatest problem in pragmatics. Preschoolers with SLI did not differ from the TD preschoolers, so they do not seem to have pragmatic difficulties according to their parents.

\section{The Role of ADHD Characteristics in Communication}

The hyperactivity and inattention characteristics obtained with the PBQ survived in almost none of the conducted regression analyses as a predictor. This implies that the score on the hyperactivity scale and the inattention scale of the PBQ did not explain a significant amount of the variance of the scores on the CCC-2 scales except for stereotyped language. This is in contrast with the findings related to the impulsivity scale. The score on this scale significantly predicted the score on almost every CCC-2 scale (see below for details).

Scores on the following CCC-2 scales were higher only when the score on the impulsivity was higher: inappropriate initiation $\left(R^{2}=.30\right)$, use of context $\left(R^{2}=.13\right)$, nonverbal communication $\left(R^{2}=.07\right)$, social relations 
$\left(R^{2}=.09\right)$, and interests $\left(R^{2}=.24\right)$. The impulsivity score was also an important predictor for most of the other scales, however, also other predictors were essential to predict the score on the rest of the CCC-2 scales.

In the speech output, syntax and coherence scale, age was a significant predictor $\left(R^{2}=.09, R^{2}=.07\right.$ and $R^{2}=.07$, respectively), but adding the PBQ impulsivity score improved the prediction of the score on these three scales $\left(R^{2}=.14, R^{2}=.14\right.$ and $R^{2}=.16$, respectively). This implies that when children became older, their speech output, syntax, and coherence abilities improved, and as the impulsivity score increased the scores on these three CCC2 scales also increased. So higher impulsivity scores predicted the presence of more language difficulties.

Age was also a significant predictor for the scores on the GenComC and the GenPragS of the CCC- $2\left(R^{2}=.06\right.$, and $R^{2}=.03$, respectively) and again more variance could be explained when adding the impulsivity score as predictor $\left(R^{2}=.21\right.$ and $R^{2}=.23$, respectively).

Although age did not affect the score on the stereotyped language scale, both the impulsivity and inattention scores were affecting the score (only impulsivity $R^{2}=.06$; both predictors $R^{2}=.11$ ). None of the included four predictors affected the score on the semantics scale.

\section{Conclusions of Study 2}

On most of the scales there was a trend for the ASD group to obtain the highest scores, followed by the SLI group, and the TD group obtained the lowest scores. Hence, according to the parents reports preschoolers with ASD showed more profound language/communication deficits than preschoolers with SLI. In contrast to Study 1 , preschoolers with ASD showed deficiencies in all CCC-2 scales, including those related to language form. In the school aged ASD children deficiencies were present in all scales except for the scales related to language form. In the older children with ASD the pragmatic/social deficiencies were disproportional to the language form deficiencies, while the opposite pattern was found in the preschoolers with ASD.

Especially impulsivity seems to have an impact on both language structure and pragmatics, while inattentiveness and hyperactivity do not. This impact is often larger than the effect of age. Please note that none of the preschoolers included in the SLI group had a clinical diagnosis of ADHD. However, this information was not available for the ASD group. As ADHD is an often reported comorbid disorder for ASD (Keen and Ward 2004; Roeyers et al. 1998) it could well be that some preschoolers in the ASD group had a comorbid diagnosis of ADHD. As we already argued it is disputable whether the diagnosis of ADHD is already a valid diagnosis in preschoolers because it is difficult to distinguish between clinically significant problem behaviors and those that are transient (Campbell 1995; Marakovitz and Campbell 1998). However, the current results suggest that impulsivity can be viewed as a predictor of and plays an important role in the existence or development of language abilities.

\section{General Discussion}

The current studies had four specific aims. First, to replicate earlier findings (Bishop and Baird 2001; Geurts et al. 2004) of similarities and differences in language profiles of skills and deficiencies in children with ASD and ADHD with the CCC-2 (Bishop 2003), the successor of the CCC (Bishop 1998). Also with the CCC-2 the pragmatic deficits in children with ADHD were evident and were with respect to these deficits indistinguishable of children with ASD. However, the deficits that children with ASD and ADHD encountered were not restricted to pragmatics as has been shown with the heightened general communication score in both groups. Differences between these two clinical groups were present in the use of context, use of non verbal communication, and their quality of social relationships. The current study shows that the CCC-2 is a valid measure to distinguish between these separable but also overlapping developmental disorders (see also Bishop 2003; Norbury et al. 2004).

The second study was conducted in order to explore whether the results of Study 1 regarding the ASD school aged group could be generalized to preschoolers. This was not the case. Preschoolers with ASD seem to have a different profile on the CCC-2 than school aged children. Besides pragmatic language problems preschoolers with ASD had deficiencies in structural aspects of language such as syntax. These types of deficits were not present in children with ASD of older age. This is in line with Rapin and Dunn (2003) who reported in their review that the pragmatic deficits seem to be more prominent relative to the structural language deficits as children with ASD become older. However, it is important to note that the CCC- 2 is a parent report and it might be that parents are more focused on structural language difficulties when children are young, while this focus changes to pragmatic abilities when children grow older. A fruitful avenue for future research would be to directly test the children on different aspects of language and communication to see whether more objective measures are in line with the parent reports. Hence, it might be that when testing the children themselves other patterns emerge.

Third, we wanted to investigate whether preschoolers with ASD can be differentiated in terms of the nature and extent of their communication problems-based on the CCC-2 parent report-from preschoolers with SLI. This was indeed the case. Children with ASD were more profoundly 
impaired than children with SLI in a number of different aspects of language. However, both clinical groups showed similar deficits in language structure. The SLI group was a very heterogeneous group in which children with a broad range of different type of language related problems were included (from stuttering to specific expressive language disorders). In this perspective it is impressive that the children with ASD could be differentiated from the children with SLI. The CCC-2 is a valid instrument to distinguish between children with language related difficulties (including ASD, SLI, and even ADHD as has been shown in Study 1) and TD children. This makes the CCC-2 an instrument that can be used for screening purposes across a broad age range (4-16 years, see also Norbury et al. 2004).

Fourth, we focused on the impact of the presence of ADHD characteristics in preschoolers on their language abilities. Especially the amount of impulsivity characteristics affects the language abilities, including language form and pragmatics. It was already known that children with ADHD often encounter all types of language deficiencies (Study 1, Baird et al. 2000; Bruce et al. 2006; Oram et al. 1999; Tannock and Schachar 1996) and that children with language disorders at early age are at risk of developing ASD or ADHD like behavior; some do even receive a psychiatric diagnosis at older age (Miniscalco et al. 2006; Snowling et al. 2006). However, it might be that both impulsivity and the language disorder(s) are already present in early development and that the combination and/or corroboration of these two leads to the emergence of a full blown developmental disorder later in life. The combination of several deficiencies in different developmental domains before the age of six might have a cascade effect during later development. As these children grew older these developmental domains are more profoundly effected. In contrast, it seems that children with ASD at older age also overcome some difficulties, as least according to their parents, as the deficits in language form seem not to be so prominent at school age. It might be that the improved language structure capacities are the effect of therapy and/or maturity, while the effect of treatment of pragmatics is less successful. This lack of improvement might be due to the more persistent character of the pragmatic problems which are at the core of the ASD diagnosis.

There are two caveats in the current study, complicating the interpretation of our findings. First, the current study focused on parent reports and did not include language measures obtained by other instruments. Second, the diagnoses of the participating children have been based on the assessment by clinical multi-disciplinary teams. These clinicians diagnosis are probably less valid than diagnosis made with standard diagnostic measures. There might have been a selection bias for clinicians to give the questionnaires to children who are expected to have communication problems, although the clinicians were not aware of the purpose of this study. However, Geurts et al. (2004) showed that the language profiles in children with ADHD and ASD based on parent ratings of the language structure and pragmatics were similar for children with a solely clinicians diagnosis as for children with a diagnosis based on extensive standard assessment procedures. Moreover, even thought there might be overlap between the different clinical groups, all children had a severe and persistent developmental disorder as all children received special educational provisions specific for these type of disorders. By including only children with a clinical diagnosis we increased the possibility that our findings would be interpretable for daily clinical practice in which the CCC-2 is widely used.

Important for the practicality of using the CCC-2 as screening instrument is the observation that (see Study 1 and Study 2) there were always a number of parents (9.3$22.8 \%$ ) that were inconsistent in their answers on the CCC2. This is most likely due to the change in questions types throughout the CCC-2. In the beginning of the CCC-2 all questions are negatively formulated (focusing on difficulties) and the last 20 questions are positively formulated (focusing on strengths). Even though the instructions clearly state that there is a change in question type, a proportion of the parents keeps answering the last questions as if they were still negatively formulated. The effect of this is that the total scores of each scales will be lower (indicating less difficulties) than when the questions were answered consistently. This implies that it is important that the clinician checks whether the parents understood the instructions clearly otherwise one might underestimate the difficulties a child encounters. This observation is especially important when using the CCC-2 in individual assessment of the communication pattern of a child, as we also showed that on a group level this inconsistent pattern of answering hardly affected the main outcomes.

The present studies add in important ways to the current literature on language skills and language deficiencies in ASD by demonstrating that parents reports of communication patterns in ASD children change over time. This implies that it is important to evaluate the communication (dis)abilities of the child regularly, in order to be able to track these changes over time and subsequently alter the focus in therapy and make adjustments in the environment of the child. Suppose that testing the children themselves reveals that they are encountering similar types of difficulties at different ages, then it is still an important finding that the perspective of the parents changes. For example when the parents themselves are worried about the pragmatic difficulties, but their therapists asks them to practice language structure skills with their children; this might not be beneficial for their cooperation and treatment fidelity. 
So, besides objective assessment of the child, it is important to know which communication difficulties are the most prominent regarding to the parents.

Moreover, the current studies imply that it is important to take into account language abilities when assessing children with ADHD, but it is also informative to include ADHD screening instruments when dealing with children with SLI or ASD. Impulsivity seems to be the most powerful and reliable predictor of the existence of communication problems. Pragmatic ability is probably affected by structural language skills, impulsivity, and autistic behavior and vice versa. Most likely these aspects constantly corroborate each other, without being able to reliable distinguishing the underlying core behavior. Therefore, focusing solely on pragmatics without taking into account other language and cognitive skills and deficiencies will not tell the complete story of the child and might, therefore, result in too narrowly defined treatment goals. Multi-disciplinary assessment and evaluations of the communication profile of a child on a regular basis are needed to be able to design an adequate and to the child tailored treatment.

Acknowledgments We gratefully acknowledge Dorothy Bishop for giving permission to translate the CCC-2 to Dutch. Furthermore, we want to thank the parents who participated in this study and the clinicians and the students who helped us with gathering all the data: Maretha de Jonghe, Peter Graauwmans, Judith de Vroomen, Koen van Loenen, Bianca Boyer, Mark Broeders, Mieke Steegs, and Johanneke Uittenbroek.

Open Access This article is distributed under the terms of the Creative Commons Attribution Noncommercial License which permits any noncommercial use, distribution, and reproduction in any medium, provided the original author(s) and source are credited.

\section{References}

Adams, C., \& Lloyd, J. (2005). Elicited and spontaneous communicative functions and stability of conversational measures with children who have pragmatic language impairments. International Journal of Language and Communication Disorders, 40(3), 333-347. doi:10.1080/13682820400027768.

American Psychiatric Association. (2000). Diagnostic and statistical manual of mental disorders (4th ed.). Washington, DC: American Psychiatric Association.

Baird, J., Stevenson, J. C., \& Williams, D. C. (2000). The evolution of ADHD: A disorder of communication? Quarterly Review Biology, 75(1), 17-35. doi:10.1086/393256.

Bara, B. G., Bosco, F. M., \& Bucciarelli, M. (1999). Developmental pragmatics in normal and abnormal children. Brain and Language, 68(3), 507-528. doi:10.1006/brln.1999.2125.

Bishop, D. V. M. (1998). Development of the Children's Communication Checklist (CCC): A method for assessing qualitative aspects of communicative impairment in children. Journal of Child Psychology and Psychiatry, 39(6), 879-891. doi: 10.1017/S0021963098002832.
Bishop, D. V. M. (2003). The Children's Communication Checklist-2. London: Psychological Corporation.

Bishop, D. V. M., \& Baird, G. (2001). Parent and teacher report of pragmatic aspects of communication: Use of the Children's Communication Checklist in a clinical setting. Developmental Medicine and Child Neurology, 43(12), 809-818. doi: 10.1017/S0012162201001475.

Bishop, D. V. M., \& Norbury, C. F. (2002). Exploring the borderlands of autistic disorder and specific language impairment: A study using standardised diagnostic instruments. Journal of Child Psychology and Psychiatry, 43(7), 917-929. doi:10.1111/ 1469-7610.00114.

Botting, N. (2003). Children's Communication Checklist (CCC) scores in 11-year-old children with communication impairments. International Journal of Language and Communication Disorders, 38, 1-13.

Botting, N., \& Conti Ramsden, G. (1999). Pragmatic language impairment without autism: The children in question. Autism, 3(4), 371-396. doi:10.1177/1362361399003004005.

Bruce, B., Thernlund, G., \& Nettelbladt, U. (2006). ADHD and language impairment: A study of the parent questionnaire FTF (Five to Fifteen). European Child \& Adolescent Psychiatry, 15(1), 52-60. doi:10.1007/s00787-006-0508-9.

Campbell, J. B. (1995). Behavior problems in preschool children: A review of recent research. Journal of Child Psychology and Psychiatry, 36, 113-149. doi:10.1111/j.1469-7610.1995.tb01657.x.

Geurts, H. M. (2007). CCC-2-NL: Children's Communication Checklist-2. Amsterdam: Harcourt Assessment B.V.

Geurts, H. M., Verte, S., Oosterlaan, J., Roeyers, H., \& Sergeant, J. A. (2004). How specific are executive functioning deficits in attention deficit hyperactivity disorder and autism. Journal of Child Psychology and Psychiatry, 45(4), 836-854. doi: 10.1111/j.1469-7610.2004.00276.x.

Gilmour, J., Hill, B., Place, M., \& Skuse, D. H. (2004). Social communication deficits in conduct disorder: A clinical and community survey. Journal of Child Psychology and Psychiatry, 45(5), 967-978. doi:10.1111/j.1469-7610.2004.t01-1-00289.x.

Keen, D., \& Ward, S. (2004). Autistic spectrum disorder. Autism, 8(1), 39-48. doi:10.1177/1362361304040637.

Kjelgaard, M. M., \& Tager Flusberg, H. (2001). An investigation of language impairment in autism: Implications for genetic subgroups. Language and Cognitive Processes, 16(2-3), 287-308. doi:10.1080/01690960042000058.

Landa, R. J. (2005). Assessment of social communication skills in preschoolers. Mental Retardation and Developmental Disabilities Research Reviews, 11(3), 247-252. doi:10.1002/mrdd.20079.

Landa, R. (2007). Early communication development and intervention for children with Autism. Mental Retardation and Developmental Disabilities Research Reviews, 13(1), 16-25. doi: 10.1002/mrdd.20134.

Lord, C., \& Paul, R. (1997). Language and communication in autism. In D. J. Cohen \& F. R. Volkmar (Eds.), Handbook of autism and pervasive developmental disorders (pp. 195-225). New York: John Wiley \& Sons, Inc.

Marakovitz, S. E., \& Campbell, S. B. (1998). Inattention, impulsivity, and hyperactivity from preschool to school age: Performance of hard-to-manage boys on laboratory measures. Journal of Child Psychology and Psychiatry, 39(6), 841-851. doi:10.1017/ S0021963098002777.

Martin, I., \& McDonald, S. (2003). Weak coherence, no theory of mind, or executive dysfunction? Solving the puzzle of pragmatic language disorders. Brain and Language, 85(3), 451-466. doi: 10.1016/S0093-934X(03)00070-1.

Mawhood, L., Howlin, P., \& Rutter, M. (2000). Autism and developmental receptive language disorder-A comparitive follow-up in early adult life. I: Cognitive and language 
outcomes. Journal of Child Psychology and Psychiatry, 41(5), $547-559$

Miniscalco, C., Nygren, G., Hagberg, B., Kadesjo, B., \& Gillberg, C. (2006). Neuropsychiatric and neurodevelopmental outcome of children at age 6 and 7 years who screened positive for language problems at 30 months. Developmenal Medicine Child Neurology, 48(5), 361-366. doi:10.1017/S0012162206000788.

Norbury, C. F., Nash, M., Baird, G., \& Bishop, D. (2004). Using a parental checklist to identify diagnostic groups in children with communication impairment: a validation of the Children's Communication Checklist-2. International Journal of Language and Communication Disorders, 39(3), 345-364. doi:10.1080/ 13682820410001654883.

Oram, J., Fine, J., Okamoto, C., \& Tannock, R. (1999). Assessing the language of children with attention deficit hyperactivity disorder: role of executive function. American Journal of SpeechLanguage Pathology, 8, 72-80.

Rapin, I. (1996). Practitioner review: Developmental language disorders: A clinical update. Journal of Child Psychology and Psychiatry, 37(6), 643-655. doi:10.1111/j.1469-7610.1996.tb01456.x.

Rapin, I., \& Dunn, M. (2003). Update on the language disorders of individuals on the autistic spectrum. Brain and Development, 25(3), 166-172. doi:10.1016/S0387-7604(02)00191-2.

Roeyers, H., Keymeulen, H., \& Buysse, A. (1998). Differentiating attention-deficit/hyperactivity disorder from pervasive developmental disorder not otherwise specified. Journal of Learning Disabilities, 31(6), 565-571.
Smidts, D. P., \& Oosterlaan, J. (2005). Gedragsvragenlijst voor kleuters [Preschool Behaviour Questionnaire]. Amsterdam: Harcourt Assessment B.V.

Snowling, M. J., Bishop, D. V., Stothard, S. E., Chipchase, B., \& Kaplan, C. (2006). Psychosocial outcomes at 15 years of children with a preschool history of speech-language impairment. Journal of Child Psychology and Psychiatry, 47(8), 759765. doi:10.1111/j.1469-7610.2006.01631.x.

Tannock, R., \& Schachar, R. (1996). Executive dysfunction as an underlying mechanism of behavior and language problems in attention deficit hyperactivity disorder. In M. M. Konstantareas, R. Tannock, J. H. Beitchman, N. J. Cohen (Ed.). (1996). Language, learning, and behavior disorders: Developmental, biological, and clinical perspectives (pp. 128 155; xv, 582 pp.). New York, NY, US: Cambridge University Press.

Thorell, L. B., \& Wahlstedt, C. (2006). Executive functioning deficits in relation to symptoms of ADHD and/or ODD in preschool children. Infant and Child Development, 15(5), 503-518. doi: 10.1002/icd.475.

Towbin, K. E., Pradella, A., Gorrindo, T., Pine, D. S., \& Leibenluft, E. (2005). Autism spectrum traits in children with mood and anxiety disorders. Journal of Child and Adolescent Psychopharmacology, 15(3), 452-464. doi:10.1089/cap. 2005.15.452.

Volkmar, F. R., Lord, C., Bailey, A., Schultz, R. T., \& Klin, A. (2004). Autism and pervasive developmental disorders. Journal of Child Psychology and Psychiatry, 45(1), 135-170. doi: 10.1046/j.0021-9630.2003.00317.x. 